Universidade Tecnológica Federal do Paraná - UTFPR Campus Ponta Grossa - Paraná - Brasil

ISSN: 1981-3686/ v. 08, n. 02: p. 1374-1384, 2014

D.O.I. $10.3895 / \mathrm{S} 1981-36862014000200007$
Revista Brasileira de Tecnologia

Agroindustrial

\title{
POLPA DE CAMU-CAMU LIOFILIZADA E ARMAZENADA EM DIFERENTES EMBALAGENS
}

\section{CAMU-CAMU PULP LYOPHILIZED AND STORED IN DIFFERENT PACKAGES}

\author{
Fernanda Francetto Juliano ${ }^{1}$, Paula Porrelli Moreira da Silva ${ }^{2}$, Renata Cristina Casemiro ${ }^{3}$, Maria \\ Helena Costa ${ }^{4}$, Marta Helena Fillet Spoto ${ }^{5}$ \\ 1, 2, 3,4,5 Universidade de São Paulo, Escola Superior de Agricultura Luiz de Queiroz - USP/ESALQ - \\ Piracicaba/SP - Brasil - pporrelli@uol.com.br
}

\begin{abstract}
Resumo
O camu-camu, fruto Amazônico, possui altos teores de ácido ascórbico além de outros importantes componentes com atividade antioxidante, a utilização desses recursos naturais pode resultar em maior desenvolvimento da região de sua origem. No Brasil, sua comercialização é limitada a frutas frescas, mas muitas pesquisas procuram produtos transformados que podem ser facilmente transportados e armazenados. O objetivo deste estudo foi avaliar a qualidade físico-química de polpa de camu-camu desidratada por liofilização e armazenada a $25{ }^{\circ} \mathrm{C}$ durante cinco meses em bolsas de polietileno, nylon e polietileno revestido com camada de alumínio. A polpa de camu-camu liofilizada embalada em bolsa de polietileno revestida com camada de alumínio foi a que obteve características satisfatórias de conservação durante os 150 dias de armazenamento, pois manteve o produto estável em virtude da menor aquisição de umidade, e da prevenção de oxidação dos pigmentos e alterações na coloração do pó.
\end{abstract}

Palavras-chave: Myrciaria dubia; amazônia; vitamina C; desidratação a frio; vida útil.

\section{Introdução}

O camu-camu (Myrciaria dubia) é um fruto silvestre pertencente à família Myrtaceae, de ocorrência nas margens de rios e lagos da Amazônia. A grande importância do fruto é devido ao seu alto valor nutricional, por ser rico em antioxidantes, como o ácido ascórbico e as antocianinas, evidenciada pela cor vermelho-púrpura do fruto (MAEDA et al., 2007; RUFINO et al., 2011).

Segundo Chirinos (2009), o conteúdo de vitamina C dos frutos criou uma demanda por essa fruta no mercado de produtos naturais. De acordo com Inoue (2008), o suco de camucamu tem elevada capacidade antioxidante e anti-inflamatória, podendo ser utilizado como suplementos dietéticos. Porém, sabe-se que o consumo dos frutos, bem como de seus 
subprodutos, ainda é reduzido devido ao fato do fruto fresco apresentar elevada acidez, além de ser altamente perecível em temperatura ambiente. Tais fatores evidenciaram a necessidade de desenvolvimento de produtos à base de camu-camu viabilizando, desta maneira, maior período de vida útil e o consumo do fruto em forma de polpa.

As tecnologias de secagem são interessantes para atender às necessidades da indústria de polpa daquele fruto, pois reduzem a utilização de energia e custos de transporte e armazenamento, além de propiciar ao consumidor um produto que se mantém viável por um intervalo de tempo duradouro. Entre estas tecnologias destacam-se a desidratação osmótica, a secagem a vácuo, a liofilização, a secagem por vapor super aquecido, bomba de calor, microondas e aspersão, que estão oferecendo grande espaço para a produção de produtos de melhor qualidade secos e em pó (SAGAR e SURESH, 2010).

Devido à ausência de água líquida e às baixas temperaturas requeridas no processo de liofilização, o encolhimento e a migração de sólidos solúveis no interior do material são minimizados, a estrutura porosa do material seco facilita a rápida reidratação de componentes aromáticos voláteis, e as reações degradativas são minimizadas, além de permitir que o produto tenha maior vida de prateleira devido à redução da atividade de água, o que é associado à redução no custo de transporte e armazenamento, devido à leveza e compactação, combinadas aos efeitos benéficos da estabilidade microbiológica e química dos alimentos (RATTI, 2001; GEORGE e DATTA, 2002; FELLOWS, 1994).

A liofilização é um processo de secagem constituído em três etapas: congelamento, secagem primária e secagem secundária. O material, previamente congelado, é desidratado por sublimação seguida pela dessorção, utilizando-se baixas temperaturas de secagem a pressões reduzidas (TATTINI JR et al., 2006). De acordo com Sablani (2006), a retenção de vitamina $\mathrm{C}$ em produtos liofilizados é significantemente maior que a de produtos secos por estufa e secos ao sol.

Contudo, a vida de prateleira de um alimento desidratado depende de fatores extrínsecos, como tamanho e propriedades da embalagem, condições ambientais de estocagem (umidade, concentração de oxigênio, luz e temperatura), transporte e manuseio, além de fatores intrínsecos, como composição química do alimento, tipo e concentração de aditivos (ALVES e BORDIN, 1998; CANO-CHAUCA, 2005; KOWALSKA e LENART, 2005).

A conservação de produtos liofilizados exige propriedades específicas das embalagens utilizadas no acondicionamento dos mesmos. De forma geral, estas embalagens devem apresentar propriedade de barreira e resistência mecânica adequadas para sua aplicação, 
dentre outras propriedades inerentes a embalagens de alimentos (VIANNA et al., 2006). Além das características necessárias citadas, em produtos liofilizados a embalagem deve oferecer barreira à luz, que pode ser obtida através de acabamento metalizado, diminuindo a degradação do produto embalado (BARUFFALDI, 1998; BOBBIO, 1992). Segundo Endo et al. (2007) afirmam que embalagens laminadas tem sido mais efetivas em relação às de polietileno no prolongamento da vida útil de sucos desidratados, conforme demonstram alguns resultados, devido à maior proteção contra a oxidação e absorção de umidade.

A fim de se possibilitar o consumo do camu-camu em forma de polpa, aumentando a sua durabilidade e manutenção de suas características originais, o presente estudo objetivou avaliar a qualidade físico-química do produto liofilizado, embalado em três diferentes embalagens (polietileno, nylon e polietileno revestido com camada de alumínio) sob armazenamento a $25^{\circ} \mathrm{C}$ durante seis meses.

\section{Material e Métodos}

\section{Processamento}

Os frutos de camu-camu foram provenientes de Cajati (SP), colhidos manualmente no mês de abril de 2011, no período da manhã, e transportados no mesmo dia para Piracicaba (SP). Foram selecionados visualmente quanto ao grau de maturação (maduros), dimensão, cor, aparência e sanidade; em seguida foi realizada a lavagem em água corrente para a retirada de sujidades. Logo após foi feita a higienização, através da imersão dos frutos em tanque de aço inoxidável contendo solução de cloro a $200 \mathrm{mg} \mathrm{L}^{-1}$ durante 15 minutos e novamente lavados em água corrente. Depois a polpa foi extraída em despolpador de aço inoxidável modelo Bonina Compacta (NPC Metalúrgica Indústria e Comércio Ltda., Itabuna, BA, Brasil), e congelada a $-18^{\circ} \mathrm{C}$ em câmara de congelamento lento nas bandejas de alumínio do liofilizador. Após este processo o produto foi liofilizado em equipamento E-C MODULYO durante 48 horas, macerado e acondicionado nas embalagens de polietileno (24 x 18 x 0,20), nylon $(24,5 \times 17,5$ x 0,20) e polietileno revestida com camada de alumínio (23 x 17 x 0,15), cedidas pela empresa Embaquim Ind. e Com. Ltda (São Paulo, SP), as quais constituíram três tratamentos.

As amostras foram armazenadas ao abrigo da luz em BOD (TECNAL - TE-391, Piracicaba, SP) a $25^{\circ} \mathrm{C}$ para controle adequado da temperatura. As análises físico-químicas, em triplicata, foram realizadas nos períodos 1, 30, 60, 90, 120 e 150 dias de armazenamento. 
Análises físico-químicas da polpa de camu-camu liofilizada

A umidade foi avaliada de acordo com AOAC (2005), os resultados foram expressos em porcentagem de polpa de camu-camu liofilizada. Avaliou-se a coloração do produto em pó liofilizado através do colorímetro Color Meter-Minolta 200b, de acordo com Minolta (1998).

A polpa de camu-camu foi reconstituída na proporção 1:9 (produto liofilizado:água) para a realização das demais análises físico-químicas: acidez titulável, determinada e calculada por titulometria com $\mathrm{NaOH} 1 \mathrm{M}$ até pH 8,1 (AOAC, 2005); antocianinas totais (AOAC, 2005); e teor de ácido ascórbico (STROHECKER, 1967).

\section{Análise estatística dos resultados}

Os resultados obtidos foram submetidos à análise de variância (ANOVA) pelo Teste $\mathrm{F}$ e comparação das médias pelo Teste de Tukey (5\%), utilizando-se o sistema estatístico Statistical Analysis System (SAS 1996). As análises estatísticas consideraram todos os períodos de armazenamento, repetições e tratamentos determinados.

\section{Resultados e Discussão}

\section{Umidade}

Em relação ao período de armazenamento, em todos os tratamentos ocorreu aumento da umidade a partir do dia 30, mantendo-se constante até o final do experimento. A polpa embalada em nylon foi a que mais adquiriu umidade no primeiro mês, com aumento de aproximadamente três vezes com relação ao valor inicial, enquanto que para os outros tratamentos tal taxa foi cerca de duas vezes (Tabela 1).

Tabela 1 - Umidade (\%) das amostras de polpa de camu-camu liofilizadas e armazenadas em diferentes períodos (valores médios, $\pm \mathrm{DP}, \mathrm{n}=3$ ).

\begin{tabular}{ccccccc}
\hline Tratamento & Dia 1 & Dia 30 & Dia 60 & Dia 90 & Dia 120 & Dia 150 \\
\hline Polietileno & $11,00 \pm 1,50 \mathrm{Ad}$ & $27,30 \pm 3,50 \mathrm{Aa}$ & $23,21 \pm 3,50 \mathrm{Aab}$ & $19,62 \pm 0,67 \mathrm{Abc}$ & $17,49 \pm 0,61 \mathrm{Ac}$ & $22,29 \pm 0,38 \mathrm{Aabc}$ \\
Nylon & $7,50 \pm 3,54 \mathrm{Ac}$ & $25,34 \pm 4,58 \mathrm{Aa}$ & $23,44 \pm 4,58 \mathrm{Aa}$ & $20,19 \pm 1,16 \mathrm{Aab}$ & $17,42 \pm 0,35 \mathrm{Ab}$ & $21,94 \pm 0,25 \mathrm{Aab}$ \\
Alumínio & $12,57 \pm 0,00 \mathrm{Ad}$ & $26,26 \pm 0,00 \mathrm{Aa}$ & $21,70 \pm 0,96 \mathrm{Aab}$ & $17,65 \pm 1,11 \mathrm{Abcd}$ & $14,95 \pm 0,23 \mathrm{Acd}$ & $20,15 \pm 0,58 \mathrm{Abc}$ \\
\hline
\end{tabular}

Nota: Média \pm Desvio Padrão, $n=3$. Letras maiúsculas iguais na coluna e minúsculas iguais na linha não diferem entre si a 5\% de probabilidade pelo Teste de Tukey.

$\mathrm{O}$ aumento da umidade do produto pode estar relacionado à falha de selagem da embalagem, bem como da alta taxa de permeabilidade das embalagens estudadas, pois as amostras foram armazenadas em ambiente de temperatura e de umidade controladas. De acordo com Cabral e Alvim (1981), conforme o tempo e as condições de umidade relativa e a 
temperatura do ambiente, pode ocorrer a migração de água para o produto, sendo esta acentuada em temperaturas mais elevadas, devido ao fato da permeabilidade dos filmes aumentar com a temperatura.

Segundo Soares et al. (2001) também observaram um aumento de umidade em pós de acerola armazenados em embalagem de polietileno laminada mostrando que, embora a permeabilidade da mesma seja baixa e recomendada pela literatura para uso em alimentos desidratados, a soldagem da mesma é difícil e pode ter comprometido o fechamento hermético da embalagem, permitindo alguma absorção de água durante o armazenamento.

Bortolatto e Lora (2008) obtiveram teor de umidade 4,04\% para abacaxi liofilizado, porém o valor de camu-camu liofilizado se aproxima do encontrado por Menezes et al. (2009) para acerola verde, que foi de 10,67\% no primeiro dia (Tabela 1).

Em açaí liofilizado e acondicionado em bolsa de polietileno revestida com folha de alumínio Menezes et al. (2008) obtiveram 4,92\% de umidade do pó, valor inferior aos encontrados em polpa de camu-camu liofilizada, o que indica elevada higroscopicidade desse produto.

\section{Cor instrumental da polpa de camu-camu liofilizada}

Houve diminuição significativa $(\mathrm{p}<0,05)$ da luminosidade $\left(\mathrm{L}^{*}\right)$ nas polpas liofilizadas acondicionadas nas embalagens de polietileno e nylon durante o período de armazenamento, evidenciando escurecimento do produto. A amostra embalada em polietileno com camada de alumínio se manteve constante durante 120 dias (Tabela 2). Tais acontecimentos podem ser justificados pelo fato da embalagem com camada de alumínio barrar a incidência de luz nas amostras, evitando, assim, a degradação dos pigmentos e a oxidação de alguns compostos presentes no produto.

Além desses, existem outros fatores que podem influenciar o escurecimento de produtos desidratados, como as reações não enzimáticas (Maillard, caramelização e oxidação de ácidos orgânicos), atividade de água alta, temperatura de armazenamento, $\mathrm{pH}$ e a composição química do alimento. A taxa de escurecimento aumenta consideravelmente quando o teor de água do produto é maior que 4 a $5 \%$ e sob temperatura de estocagem acima de $38{ }^{\circ} \mathrm{C}$ (SALUNKHE et al., 1991; FELLOWS, 2006).

Resultado semelhante foi encontrado por Endo et al. (2007), que ao avaliarem a vida de prateleira de maracujá desidratado por aspersão, verificaram que a luminosidade do produto foi reduzida durante o período de armazenamento. 
Todos os tratamentos apresentaram decréscimo do ângulo Hue, tendendo do rosado para o vermelho alaranjado até o dia 60, com elevação no dia 90 e posterior decréscimo até o final do período de armazenamento. Já entre os tratamentos, apenas no dia 30 a polpa liofilizada embalada em polietileno apresentou diferença significativa $(\mathrm{p}<0,05)$ e no dia 60 apenas a amostra embalada em alumínio teve esse comportamento. Durante o período de armazenamento todas as amostras apresentaram alteração na coloração, porém, a polpa liofilizada embalada em bolsa de polietileno com camada de alumínio foi a que se modificou em menor intensidade (Tabela 2).

As polpas liofilizadas embaladas em bolsas de polietileno e nylon apresentaram valor máximo de cromaticidade (Croma) no dia 30, indicando que a coloração das amostras estava mais saturada. O valor croma em todas as polpas liofilizadas aumentou no dia 90 e mantevese constante até o final do experimento. Já em relação aos tratamentos, o croma foi estatisticamente diferente apenas no dia 120 (Tabela 2).

As variações encontradas tanto para o ângulo de cor Hue quanto para cromaticidade nas amostras de polpa de camu-camu liofilizada, pode ser justificada, também, pela oxidação dos pigmentos presentes, ação da luz (principalmente nas embalagens de polietileno e nylon) e também, pela absorção de água e oxigênio do ambiente.

Acidez titulável, Antocianinas totais e Teor de ácido ascórbico

\section{Acidez titulável (\% em ácido cítrico)}

A acidez titulável da polpa reconstituída de camu-camu liofilizada não foi alterada estatisticamente $(\mathrm{p}<0,05)$ durante o período de armazenamento e entre os tratamentos (Tabela 3). Contudo, tais resultados diferem dos encontrados por Menezes et al. (2009) em pó de acerola obtido por liofilização, que notaram diminuição no teor da acidez quando comparado o valor final com o tempo zero de armazenamento. De acordo com esses resultados, foi possível concluir que o aumento do teor de umidade ocorrido durante o armazenamento não influenciou a acidez das amostras.

O teor de acidez titulável do produto apresentou-se semelhante ao encontrado por Peuckert et al. (2010) e também por Vieira et al. (2010) para camu-camu $(2,176 \%$ e $2,17 \mathrm{~g}$ $100 \mathrm{~mL}^{-1}$ ) respectivamente (Tabela 3 ). 
Tabela 2 - Luminosidade (L*), Ângulo Hue (graus) e Cromaticidade das amostras de polpa de camu-camu liofilizada e armazenadas em diferentes períodos (valores médios, \pm $\mathrm{DP}, \mathrm{n}=3$ ).

\begin{tabular}{|c|c|c|c|c|c|c|c|}
\hline & Trat & Dia 1 & Dia 30 & Dia 60 & Dia 90 & Dia 120 & Dia 150 \\
\hline \multirow{3}{*}{$\mathrm{L}^{*}$} & Polietileno & $53,37 \pm 2,12 \mathrm{Aa}$ & $45,91 \pm 2,69 \mathrm{Aab}$ & $44,08 \pm 4,07 \mathrm{Aab}$ & $43,22 \pm 3,19 \mathrm{Ab}$ & $39,04 \pm 4,21 \mathrm{Ab}$ & $36,67 \pm 3,76 \mathrm{Ab}$ \\
\hline & Nylon & $52,62 \pm 1,56 \mathrm{Aa}$ & $46,64 \pm 3,95 \mathrm{Aab}$ & $47,82 \pm 3,08$ Aabc & $41,82 \pm 2,81 \mathrm{Abc}$ & $37,71 \pm 2,87 \mathrm{Ac}$ & $37,24 \pm 5,47 \mathrm{Ac}$ \\
\hline & Alumínio & $52,01 \pm 1,67 \mathrm{Aa}$ & $45,61 \pm 3,99 \mathrm{Aab}$ & $43,73 \pm 1,71 \mathrm{Aab}$ & $46,27 \pm 0,74 \mathrm{Aab}$ & $43,41 \pm 1,23 \mathrm{Aab}$ & $38,98 \pm 4,41 \mathrm{Ab}$ \\
\hline \multirow{3}{*}{ Hue } & Polietileno & $52,11 \pm 1,90 \mathrm{Aa}$ & $45,79 \pm 1,15 \mathrm{Ab}$ & $20,97 \pm 1,72 \mathrm{Be}$ & $35,75 \pm 1,53 \mathrm{Abc}$ & $31,34 \pm 0,69$ Acd & $28,22 \pm 0,82 \mathrm{Ad}$ \\
\hline & Nylon & $51,12 \pm 1,68 \mathrm{Aa}$ & $39,27 \pm 1,76 \mathrm{Bb}$ & $19,76 \pm 0,45 \mathrm{Be}$ & $33,83 \pm 2,05 \mathrm{Ac}$ & $30,49 \pm 2,44$ Acd & $28,41 \pm 2,22 \mathrm{Ad}$ \\
\hline & Alumínio & $50,77 \pm 1,78 \mathrm{Aa}$ & $39,27 \pm 3,26 \mathrm{Bb}$ & $28,63 \pm 1,56 \mathrm{Ad}$ & $34,90 \pm 0,71 \mathrm{Abc}$ & $32,03 \pm 0,31$ Acd & $30,21 \pm 0,97 \mathrm{Acd}$ \\
\hline \multirow{3}{*}{ Croma } & Polietileno & $29,64 \pm 0,88 \mathrm{Ac}$ & $39,03 \pm 1,36 \mathrm{Ab}$ & $30,31 \pm 1,54 \mathrm{Ac}$ & $60,02 \pm 1,25 \mathrm{Aa}$ & $60,32 \pm 0,79 \mathrm{Aa}$ & $59,01 \pm 0,32 \mathrm{Aa}$ \\
\hline & Nylon & $29,42 \pm 0,20 \mathrm{Ac}$ & $43,08 \pm 0,63 \mathrm{Ab}$ & $29,98 \pm 1,21 \mathrm{Ac}$ & $58,97 \pm 1,45 \mathrm{Aa}$ & $58,63 \pm 2,62 \mathrm{ABa}$ & $58,99 \pm 1,53 \mathrm{Aa}$ \\
\hline & Alumínio & $30,24 \pm 0,28 \mathrm{Abc}$ & $42,62 \pm 1,59 \mathrm{Ab}$ & $28,79 \pm 1,98 \mathrm{Ac}$ & $55,48 \pm 0,84 \mathrm{Aa}$ & $54,71 \pm 1,35 \mathrm{Ba}$ & $56,48 \pm 3,62 \mathrm{Aa}$ \\
\hline
\end{tabular}

Nota: Média \pm Desvio Padrão, $\mathrm{n}=3$. Letras maiúsculas iguais na coluna e minúsculas iguais na linha não diferem entre si a $5 \%$ de probabilidade pelo Teste de Tukey.

Tabela 3 - Acidez titulável (\% em ácido cítrico), Antocianinas totais em mg equivalente cyanidin-3-glucosideo. g ${ }^{-1}$ e Teor de ácido ascórbico em g 100 g-1 de ácido gálico da polpa de camu-camu liofilizada reconstituída (valores médios, $\pm \mathrm{DP}, \mathrm{n}=3$ ).

\begin{tabular}{|c|c|c|c|c|c|c|c|}
\hline & Trat & Dia 1 & Dia 30 & Dia 60 & Dia 90 & Dia 120 & Dia 150 \\
\hline & Polietileno & $2,86 \pm 0,21$ & $2,76 \pm 0,06$ & $2,73 \pm 0,00$ & $2,36 \pm 0,06$ & $2,32 \pm 0,00$ & $2,35 \pm 0,06$ \\
\hline \multirow{3}{*}{$\begin{array}{l}\text { Acidez } \\
\text { titulável }\end{array}$} & Nylon & $2,99 \pm 0,12$ & $2,73 \pm 0,17$ & $2,73 \pm 0,17$ & $2,42 \pm 0,10$ & $2,42 \pm 0,00$ & $2,32 \pm 0,00$ \\
\hline & Alumínio & $3,1 \pm 0,12$ & $2,79 \pm 0,15$ & $2,76 \pm 0,06$ & $2,56 \pm 0,06$ & $2,52 \pm 0,10$ & $2,69 \pm 0,83$ \\
\hline & Polietileno & $12,04 \pm 0,63 \mathrm{Aab}$ & $12,38 \pm 6,63 \mathrm{Aa}$ & $5,10 \pm 2,93 \mathrm{Bb}$ & $<0,0001$ & $<0,0001$ & $<0,0001$ \\
\hline \multirow[t]{3}{*}{ Antocianinas } & Nylon & $12,11 \pm 1,39 \mathrm{Aa}$ & $7,97 \pm 1,27 \mathrm{Aab}$ & $4,03 \pm 0,50 \mathrm{Bb}$ & $<0,0001$ & $<0,0001$ & $<0,0001$ \\
\hline & Alumínio & $12,22 \pm 1,16$ Aab & $11,56 \pm 2,40 \mathrm{Aab}$ & $12,62 \pm 1,95 \mathrm{Aa}$ & $3,05 \pm 0,93 \mathrm{c}$ & $5,21 \pm 0,42 \mathrm{bc}$ & $1,18 \pm 0,40 \mathrm{c}$ \\
\hline & Polietileno & $3,90 \pm 0,11 \mathrm{Aa}$ & $2,22 \pm 0,01 \mathrm{Ab}$ & $2,23 \pm 0,04 \mathrm{Bb}$ & $<0,0001$ & $<0,0001$ & $<0,0001$ \\
\hline \multirow[t]{2}{*}{ Ácido Ascórbico } & Nylon & $3,49 \pm 0,13 \mathrm{Ba}$ & $2,15 \pm 0,00 \mathrm{Ac}$ & $2,60 \pm 0,19 \mathrm{Ab}$ & $<0,0001$ & $<0,0001$ & $<0,0001$ \\
\hline & Alumínio & $4,09 \pm 0,04 \mathrm{Aa}$ & $2,13 \pm 0,03 \mathrm{Ac}$ & $2,51 \pm 0,05 \mathrm{ABb}$ & $<0,0001$ & $<0,0001$ & $<0,0001$ \\
\hline
\end{tabular}

Revista Brasileira de Tecnologia Agroindustrial 
Nota: Médias \pm Desvio padrão, $\mathrm{n}=3$. Letras maiúsculas iguais na coluna e minúsculas iguais na linha não diferem entre si a 5\% de probabilidade pelo Teste de Tukey. 
Antocianinas totais (mg equivalente cyanidin-3-glucosideo $\mathrm{g}^{-1}$ polpa reconstituída)

As polpas liofilizadas embaladas em bolsas de polietileno e nylon, a partir do dia 90 não mais apresentaram teores de antocianinas quantificáveis pela metodologia adotada (Tabela 3).

A amostra embalada em polietileno apresentou aumento no teor das antocianinas totais no dia 30 e notável declínio a partir do $60^{\circ}$ dia de armazenamento. A polpa liofilizada embalada em nylon teve seu teor de antocianinas reduzido durante todo o período de armazenamento. A amostra embalada em alumínio foi a que mais teve preservados tais pigmentos, mantendo-se estável até o dia 60, com redução aos 90. Com relação aos tratamentos, apenas no dia 60 a polpa liofilizada e embalada em alumínio apresentou maior teor de antocianinas que as demais (Tabela 3).

De acordo com Tonon et al. (2010), a taxa de degradação de antocianinas em produtos em pó pode ser atribuída ao contato do material com o oxigênio exterior ou de dentro dos poros. Uma maior absorção de água no início do armazenamento também pode ser responsável pela maior taxa de degradação, pois teores de umidade mais elevados implicam em maior mobilidade molecular. Esta justificativa está ligada à rápida degradação das antocianinas em polpa de camu-camu em pó embalado nas bolsas de polietileno e nylon, pois as suas umidades estavam acima de $25 \%$ logo no primeiro mês de armazenamento.

Ademais, a temperatura de armazenamento das amostras $\left(25^{\circ} \mathrm{C}\right)$ pode ter auxiliado na degradação das antocianinas presentes, pois estes pigmentos são termossensíveis, e aliados à presença de açúcares e proteínas podem resultar na reação de escurecimento não enzimático, que geralmente ocorre durante a armazenagem de alimentos por muito tempo (TONON et al., 2010).

Os teores de antocianinas quantificados no primeiro dia de análise diferem dos encontrados

por Rufino et al. (2011), que obtiveram 42,2 mg antocianinas totais $100 \mathrm{~g}^{-1}$ de matéria seca. Já Maeda et al. (2006), detectaram 9,98 mg antocianinas totais $100 \mathrm{~g}^{-1}$ de polpa, valores que se aproximam aos encontrados no presente estudo.

Maeda et al. (2007) também observaram redução das antocianinas durante o tempo de armazenamento, comprovando que estes pigmentos, no camu-camu, tendem a reduzir com o período de estocagem (Tabela 3). Tal fato pode ser decorrente das condições de luminosidade, pois foi observada diferença entre as amostras protegidas por película de alumínio e as sem proteção à luz. Foi notada, ainda, redução significativa do teor de antocianinas para todos os tratamentos de acordo com o período de armazenamento.

\section{Teor de ácido ascórbico ( $m g$ de ácido cítrico 100 g-1 de polpa reconstituída)}

A partir do dia 60, não foi possível quantificar o teor de ácido ascórbico através da metodologia utilizada. A polpa de camu-camu liofilizada e embalada em bolsa de polietileno apresentou declínio no dia 30 e se manteve constante até o $60^{\circ}$ dia de armazenamento. Já as 
amostras embaladas em nylon e alumínio, apresentaram declínio no dia $30 \mathrm{com}$ posterior aumento no dia 60 (Tabela 3).

Com relação aos tratamentos, no primeiro dia a polpa liofilizada embalada em nylon apresentou teor inferior aos demais tratamentos, e no dia 60 o teor de ácido ascórbico da polpa liofilizada embalada em polietileno diferiu-se da polpa liofilizada embalada em nylon $(\mathrm{p}<0,05)$, sendo esta, a que apresentou teor mais elevado deste atributo (Tabela 3).

Os valores encontrados no início do experimento são superiores aos encontrados por Maeda et al. (2006) em polpa de camu-camu, os quais quantificaram 2.585,4 mg ácido ascórbico $100 \mathrm{~g}^{-1}$ de polpa. Peuckert et al. (2010) e Chirinos et al. (2009) encontraram valores $2.641,8 \mathrm{mg} 100 \mathrm{~g}^{-1} \mathrm{e}$ $2.010 \mathrm{mg} 100 \mathrm{~g}^{-1}$, respectivamente, para o fruto de camu-camu. Just et al. (2000) quantificaram a

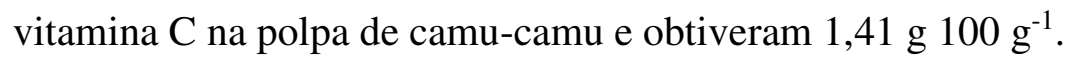

A redução do teor de ácido ascórbico verificada durante o período de armazenamento de camu-camu liofilizado concorda com o encontrado por Maeda et al. (2007) para néctar de camucamu, porém estes observaram que para os néctares armazenados em temperatura de refrigeração não houve redução dos teores (Tabela 3).

Em pó de acerola verde obtido por liofilização, Menezes et al. (2009) verificaram que o processo diminuiu o teor de vitamina $\mathrm{C}$ do produto em $33,47 \%$ durante 180 dias de armazenamento.

\title{
4 Conclusões
}

A polpa de camu-camu liofilizada embalada em bolsa de polietileno revestida com camada de alumínio foi a que obteve características satisfatórias de conservação durante os 150 dias de armazenamento, pois manteve o produto estável em virtude da menor aquisição de umidade, e da prevenção de oxidação dos pigmentos e alterações na coloração do pó.

\section{Agradecimentos}

Os autores agradecem à Fundação de Amparo à Pesquisa do Estado de São Paulo - FAPESPpelo financiamento do experimento.

\begin{abstract}
Camu-camu is a fruit that has high levels of ascorbic acid, and other important components with antioxidant activity and the use of natural resources can result in further development of the region of origin. In Brazil, the market is limited to fresh fruit, but a lot of research looking for processed products which can be easily transported and stored. The aim of this study was to evaluate the physico-chemical quality of camu-camu pulp dehydrated by lyophilization and stored at $25{ }^{\circ} \mathrm{C}$ for five months in polyethylene, nylon and polyethylene bags coated aluminum layer. The pulp of camucamu freeze dried packaged in polyethylene bag coated with aluminum layer was that obtained satisfactory characteristics of conservation during 150 days of storage because product remained stable due to lower acquisition moisture, and prevented pigment changes in color of the powder.
\end{abstract}


Key-words: Myrciaria dubia, Amazon, vitamin C, cold dehydration, shelf life.

\section{Referências}

ALVES, R. M.; BORDIN, M. R. Estimativa da vida útil de café solúvel por modelo matemático. Ciência e Tecnologia de Alimentos, Campinas-SP, v. 18, n. 1, p.19-24, 1998.

AOAC - ASSOCIATION OF OFFICIAL ANALITICAL CHEMISTRY. 2005. Official methods of analysis of the Association of Analytical Chemists International. 18.ed. Washington: AOAC, 2005.

BARUFFALDI, R. Fundamentos de Tecnologia de Alimentos, v.3. São Paulo: Ed. Atheneu, 1998.

BOBBIO, P. A.; BOBBIO, F.O. Química do Processamento de Alimentos. São Paulo: Editora Varela, 1992.

BORTOLATTO, J.; LORA, J. Avaliação da composição centesimal do abacaxi (Ananas comosus (1.) Merril) liofilizado e in natura. Revista de Pesquisa e Extensão em Saúde, Criciúma-SC, v.4, n.1, 2008.

CABRAL, A. C. D.; ALVIM, D. D. Alimentos desidratados - conceitos básicos para sua embalagem e conservação. Boletim do Instituto de Tecnologia de Alimentos, Campinas-SP, v. 1, n. 18, p. 1-65, 1981.

CANO-CHAUCA, M., STRINGHETA, P. C., RAMOS, A. M., Cal-Vidal, J. Effect of the carriers on the microstructure of mango powder obtained by spray drying and its functional characterization. Innovative Food Science and Emerging Technologies, Amsterdam, v.5, n.4, p. 420-428, 2005. http://dx.doi.org/10.1016/j.ifset.2005.05.003

CHIRINOS, R.; GALARZA, J.; BETALlELUZ-PALlARDEL, I.; PEDRESCHI, R.; CAMPOS, D. Antioxidant compounds and antioxidant capacity of Peruvian camu camu (Myrciaria dubia (H.B.K.) McVaugh) fruit at different maturity stages. Food Chemistry, Barking, v.120, p.1019-1024, 2009.

ENDO, E.; BORGES, S.V.; DAIUTO, E.R.; CEREDA, M.P.; AMORIM, E. Avaliação da vida de prateleira do suco de maracujá desidratado. Ciência e Tecnologia de Alimentos, Campinas, v.27, n.2, p.382-386, 2007. http://dx.doi.org/10.1590/S0101-20612007000200029

FELLOWS, P. Tecnología del procesado de los alimentos: Principios y prácticas. Zaragoza (España): Editorial Acribia, S.A., 1994. p.549.

FELLOWS, P. J. Tecnologia do processamento de alimentos: princípios e prática. 2. ed. Porto Alegre: Artmed, 2006, 602p.

GEORGE, J.P. \& DATTA, A. K. Development and validation of heat and mass transfer models for freeze-drying of vegetable slices, Journal of Food Engineering, London, v. 52, n.1, p. 89-93, 2002. http://dx.doi.org/10.1016/S02608774(01)00091-7

INOUE, T.; KOMODA, H.; UCHIDA,T.; KOICHI, N. Tropical fruit camu-camu (Myrciaria dubia) has anti-oxidative and anti-inflammatory properties. Journal of Cardiology, v.52, n.2, p.127-132, 2008. http://dx.doi.org/10.1016/j.jjcc.2008.06.004

JUST, K.C.; VISENTAINER, J.V.; SOUZA, E.N.; MATSUSHITA, M. Nutritional composition and vitamin C stability in stored camu-camu (Myrciaria dubia) pulp. Organo Oficial de la Sociedad Latinoamericana de Nutrición. v.50, n.4, p.405-408, 2000.

KOWALSKA, J.; LENART, A. The influence of ingredients distribution on properties of agglomerated cocoa products. Journal of Food Engineering, Oxford, v.68, n.2, p.155-161, 2005. http://dx.doi.org/10.1016/j.jfoodeng.2004.05.028

MAEDA, R.N.; PANTOJA, L.; YUYAMA, L.K.O.; CHAAR, J.M. Determinação da formulação e caracterização do néctar de camu-camu (Myrciaria dubia Mcvaugh). Ciência e Tecnologia de Alimentos, Campinas, v.26, n.1, p.70-74, 2006. http://dx.doi.org/10.1590/S0101-20612006000100012

MAEDA, R.N.; PANTOJA, L.; YUYAMA, L.K.O.; CHAAR, J.M. Estabilidade de ácido ascórbico e antocianinas em néctar de camu-camu (Myrciaria dubia (H. B. K.) McVaugh). Ciência e Tecnologia de Alimentos, Campinas, v.27, n.2, p. 313-316, 2007. http://dx.doi.org/10.1590/S0101-20612007000200018 
MENEZES, E.M.S.; TORRES, A.T.; SRUR, A.U. Valor nutricional da polpa de açaí (Euterpe oleracea Mart) liofilizada. Acta Amazonica, Manaus, v.38, n.2, p.311-316, 2008. http://dx.doi.org/10.1590/S004459672008000200014

MENEZES, A.R.V.; SILVA, A.J.; CRUZ, H.L.L.; ARAUJO, D.R.; SAMPAIO, D.D. Estudo comparativo do pó da acerola verde (Malphigia emarginata d.c) obtido em estufa por circulação de ar e por liofilização. Revista Brasileira de Produtos Agroindustriais, Campina Grande -PB, v.11, n.1, p.169-174, 2009.

MINOLTA, K. 1998. Comunicação precisa da cor: controle de qualidade da percepção à instrumentação. Osaka. 59p.

PEUCKERT, Y.P.; VIEIRA, V.B.; HECKTHEUER, L.H.R.; MARQUES, C.T.; ROSA, C.S. Caracterização e aceitabilidade de barras de cereais adicionadas de proteína texturizada de soja e camu - camu (Myrciaria dúbia). Alimentos e Nutrição, Araraquara-SP, v.21, n.1, p. 147-152, 2010.

RATTI, C. Hot air and freeze-drying of high-value foods: a review, Journal of Food Engineering, London, v.49, p.11319, 2001.

RUFINO, M.S.M.; ALVES, R.E; FABIANO, A.N.F.; EDY, S.B. Free radical scavenging behavior of ten exotic tropical fruits extracts. Food Research International, Essex, v.44, p.2072-2075, 2011. http://dx.doi.org/10.1016/j.foodres.2010.07.002

SABLANI, S.S. Drying of fruits and vegetables: retention of nutritional/ functional quality. Drying Technology, Monticello, v.24, p. 428-432, 2006.

SAGAR, V.R.; SURESH, K.P. Recent advances in drying and dehydration of fruits and vegetables: a review. Journal of Food Science and Technology, Mysore, v.47, n.1, p.15-26. 2010. http://dx.doi.org/10.1007/s13197-010-0010-8

SALUNKE, D.K.; BOLIN, H.R.; REDDY, N.R. Dehydration. In: Storage, processing and nutritional quality of fruits and vegetables, 2nd ed., Vol II, Processed fruits and vegetables, Boca Raton: CRC Press Inc., p 49-98, 1991.

SOARES, E.C.; OLIVEIRA, G.S.F; MAIA, G.A.; MONTEIRO, J.C.S.; SILVA Jr. A.; FILHO, M.S.S. Desidratação da polpa de acerola (Malpighia emarginata D. C.) pelo processo "Foam-mat". Ciência e Tecnologia de Alimentos, Campinas, v.2, n.21, p.164-170, 2001. http://dx.doi.org/10.1590/S0101-20612001000200008

STATISTICAL ANALISIS SYSTEM INSTITUTE. SAS/QC software: usage and reference (version 6). 2th ed. Cary, 1996. 1CDROM.

STROHECKER, R.; HENNING, H. M. 1967. Analisis de vitaminas: métodos comprobados. Madrid: Paz Montalvo, p.468.

TATTINI, J.R.V.; PARRA, D.F.; PITOMBO, R.N.M. Influência da taxa de congelamento no comportamento físicoquímico e estrutural durante a liofilização da albumina bovina. Revista Brasileira de Ciências Farmacêutica, São Paulo-SP, v.42, n.1, p.127-136, 2006.

TONON, R.V.; BRABET, C.; HUBINGER, M.D. Anthocyanin stability and antioxidant activity of spray-dryed acai (Euterpe oleracea) juice produced with different carrier agents. Food Research International, Essex, v.43, p.907-914, 2010. http://dx.doi.org/10.1016/j.foodres.2009.12.013

VIANNA, R.I.; MÄHLMANN, C.M.; RODRÍGUEZ, A.L.; BACCAR, N.M.; MARQUARDT, L.; ROHLFES, A.L.B. Estudo de embalagens flexíveis monocamada para acondicionamento de produtos liofilizados. Congresso Brasileiro de Engenharia e Ciência dos Materiais, Foz do Iguaçu, PR, Brasil, Novembro de 2006.

VIERA, V.B.; RODRIGUES, J.B.; BRASIL, C.C.B.; ROSA, C.S. Produção, caracterização e aceitabilidade de licor de camu-camu (Myrciaria dúbia (H.B.K.) Mcvaugh). Alimentos e Nutrição, Araraquara, v.21, n.4, p.519-522, 2010.

Submetido em 11 jun. 2013; Aceito para publicação em 14 abr. 2014. 